Daniel J. Weiner - Aviva Katz $\cdot$ Ronald B. Hirschl

Robert Drongowski $\cdot$ Arnold G. Coran

\title{
Interval appendectomy in perforated appendicitis
}

\begin{abstract}
The treatment of the perforated appendix remains controversial, with the optimal timing of surgical intervention unclear. Previous studies have documented an increase in both minor and major complication rates in patients undergoing appendectomy for perforated appendicitis. We sought to evaluate the nonoperative therapy of perforated appendicitis followed by interval appendectomy. The charts of all children undergoing admission for appendicitis during a 10 -year period $(n=480)$ were reviewed. Data were abstracted regarding patient presentation, laboratory and radiologic findings, operative and pathology reports, and postoperative course in those patients with perforated appendicitis $(n=104)$. Comparisons were made between patients undergoing primary appendectomy for perforated appendicitis $(\mathrm{n}=87)$ and those treated with IV antibiotics and hydration and then scheduled for interval appendectomy 4 to 6 weeks following the acute event $(\mathrm{n}=17)$. Treatment assignment was determined by the attending pediatric surgeon in a non-randomized fashion. No significant differences were seen between these two groups in days of antibiotic treatment, nasogastric decompression, and IV hydration. Additionally, total hospital days and cost did not differ significantly between the two groups (primary $=10.3$ days and $\$ 10,550$; interval $=13.3$ days and $\$ 13,221, P=0.11$ and 0.21 , respectively). The overall complication rates, $12.6 \%$ in the primary group and $5.9 \%$
\end{abstract}

Presented at the Surgical Section, American Academy of Pediatrics, Washington, DC, USA, 30 October-4 November 1993

D. J. Weiner

Department of Pediatrics, University of Michigan, Ann Arbor, MI, USA

R. B. Hirschl - R. Drongowski - A. G. Coran

Section of Pediatric Surgery, University of Michigan, USA

A. Katz

Thomas Jefferson University Hospital, Philadelphia, PA, USA

A. Katz (区)

Department of Surgery, A. I. duPont Institute, P. O. Box 269 ,

Wilmington, DE 19803, USA in the interval group, also did not differ significantly, while the major complication rate (wound dehiscence, abscess, and small-bowel obstruction), $10 \%$ versus $0 \%$, was significantly higher in the primary group as compared with the interval group. Our data demonstrate no significant disadvantage, and possibly an improvement in the major complication rate, with nonoperative treatment of perforated appendicitis followed by interval appendectomy. We suggest that this treatment modality should be considered when evaluating the child with perforated appendicitis.

Key words Appendicitis - Perforation - Management Child

\section{Introduction}

Appendicitis remains the most common indication for acute abdominal surgery in the pediatric population in the Western world. The overall incidence of perforated appendicitis has remained remarkably constant at approximately $30 \%-45 \%$ [11]. There have been numerous studies of the etiology of this relatively high perforation rate in children in an attempt to decrease the incidence of complicated appendicitis and its associated morbidity. The most consistent finding has been that complicated (gangrenous or ruptured appendicitis with or without associated abscess formation) appendicitis is related to delay in presentation to the surgeon rather than specific physiologic differences in the young child $[5,11,12,16]$.

These studies agree that increased duration of symptoms is the factor most responsible for increased severity of disease. One study has even raised the concern that changes in health care delivery, specifically, the development of systems utilizing "gatekeepers" to regulate patients' access to specialists, has resulted in delay of presentation to the surgeon with an associated increase in the incidence and severity of complications [12]. This is of significant concern given the current direction of the national health care environment. In contrast to the danger 
of prolonged observation by either parents or primary physicians, there has been demonstration of the safety of inpatient, surgeon-supervised observation of the child with abdominal pain to increase diagnostic accuracy without an associated increase in the incidence of perforated appendicitis [3].

It appears that a decrease in the rate of perforation will continue to be dependent on factors outside the surgeon's control - the need for further education of parents and primary care physicians in the need for timely, accurate evaluation of the child with persistent abdominal pain [11]. In contrast, efforts can be directed to the provision of safe and effective care of the child with perforated appendicitis within an overall plan of most efficient use of health resources. Previous reviews have analyzed the outcome of perforated versus nonperforated acute appendicitis cared for by a similar treatment protocol $[5,13,17,18]$. Additionally, there has been interest in the conservative or initially nonsurgical management of the patient presenting late with an appendiceal mass $[7,8,19]$. However, relatively few data have been presented in the literature on the safety and practicality of conservative management of the child presenting with perforated appendicitis not yet associated with a palpable abdominal mass $[1,15]$. Our experience with the conservative management of perforated appendicitis demonstrates the safety of this approach, with a decreased risk of complications without a significant increase in either hospital stay or patient medical costs.

\section{Materials and methods}

The charts of all children undergoing admission for acute appendicitis during a 10-year period, 1979-1989 (n = 480), were reviewed. Children undergoing incidental appendectomy were excluded. Data were abstracted regarding clinical presentation, laboratory and radiologic findings, operative and pathology reports, and postoperative hospital course in those patients with perforated appendicitis $(n=104$ $21 \%$ ). Comparisons were made between patients undergoing primary appendectomy for perforated appendicitis $(n=87)$ and those treated conservatively with IV antibiotics and hydration and then scheduled for interval appendectomy 4 to 6 weeks following the acute event $(n=17)$. All patients were evaluated prior to assignment to treatment groups by either a pediatric surgery fellow or an attending pediatric surgeon. Assignment to one of these two treatment groups was determined by the supervising surgeon in a non-randomized fashion. Determination of appendiceal perforation nonoperatively was made on the basis of the individual surgeon's clinical judgement, relying heavily on clinical presentation, duration of symptoms, and, in the later years of this review, preoperative ultrasonographic (US) findings.

Although we felt there was adequate support for the conservative management of perforated appendicitis, based on both clinical experience and a review of the literature, conservative management of acute nonperforated appendicitis was not viewed as acceptable. Therefore, if any concern was raised during the initial patient evaluation or early observation period regarding the severity of disease, the patient underwent early operative intervention in an attempt to avoid nonoperative treatment of early appendicitis. This obviously resulted in some patients with perforated appendicitis undergoing early operative intervention. All therapy was provided without regard to insurance status and after obtaining informed consent from the patient's parents or legal guardians.

All appendectomies were performed under the direct supervision of the pediatric surgery fellow or attending pediatric surgeon. The operative approach was not completely standardized, but generally included debridement of all gross fibrinous exudate and local saline irrigation, inversion of the appendiceal stump, and primary subcuticular closure of the transverse right lower quadrant incision, with minimal variation dependent on operative findings. All patients received perioperative broad-spectrum IV antibiotic therapy, including coverage for anaerobic organisms. Most recently, this therapy was standardized to include ampicillin $100 \mathrm{mg} / \mathrm{kg}$, gentamycin $5 \mathrm{mg} / \mathrm{kg}$, and metronidazole $30 \mathrm{mg} / \mathrm{kg}$ per day. Statistical analysis was completed by computer-assisted ANOVA with significance set at $P<.05$ and statistically significant differences between patient groups noted in the text.

\section{Results}

There was no mortality associated with perforated appendicitis during the 10 years of this study. Additionally, all patients were confirmed to have had appendicitis. Acute perforated appendicitis was noted in all patients undergoing primary appendectomy and scarring and fibrosis consistent with previous appendicitis in the interval appendectomy group. The two study populations were comparable, with no significant differences in age (9.2 years for the primary vs. 9.4 years for the interval group), preoperative white blood cell count (primary $=18.218$ vs. interval $=17,171$ ), or bandemia (primary $=69 \%$ vs. interval $=72 \%$, but a significant difference in the number of days of abdominal pain prior to initiation of treatment (primary $=2.7$ days, interval $=5.1$ days $)$.

Recovery time from either early appendectomy for or conservative management of perforated appendicitis was similar, with no significant difference between the two groups in the number of febrile days (primary $=2.4$ vs. interval $=2.9$ ), number of days without oral intake (primary $=3.2$ vs. interval $=2.2$ ), number of days of nasogastric decompression (primary $=2.7 \mathrm{vs}$. interval $=1.5$ ), or number of days requiring IV hydration (primary $=5 \mathrm{vs}$. interval $=4$ ) following the institution of treatment. Treatment needs of the two groups were similar, with no significant differences in total IV antibiotic usage (primary $=9.1$ days vs. interval $=11.2$ days).

The patients undergoing conservative management followed by interval appendectomy required two hospital admissions, while most of the patients in the primary appendectomy group required a single admission. Therefore, we have evaluated data regarding treatment and costs as a single total number reflecting all related hospitalizations. Despite this, there was no significant difference in total hospital days (primary $=10.3$ vs. interval $=13.2$ ) between the group treated with early operative intervention and those managed conservatively. Similarly, there was no significant difference in total hospital costs between the two groups (primary $=\$ 10,550$ vs. interval $=\$ 13,221$ ).

We had initially pursued conservative management of the perforated appendix out of concern for the relatively high morbidity still associated with this disease. We hypothesized that interval appendectomy undertaken after resolution of the initial intra-abdominal inflammatory response would be associated with less postoperative 
complications and associated morbidity. We found no significant difference in the overall complication rate between these two patient groups, $(12.6 \%$ in those undergoing early operative intervention vs. $5.9 \%$ in those managed conservatively). In contrast, when the major complications (intra-abdominal abscess, small-bowel obstruction, wound dehiscence) were analyzed as a distinct group, the $10 \%$ major complication rate in the primary group was significantly greater than the $0 \%$ noted in the group managed conservatively.

\section{Discussion}

Our perforation rate of $21 \%$ fits well within the reported perforation rate for appendicitis in childhood, with rates of $24 \%$ to $39 \%$ cited from multiple centers $[1,5,13,16,17]$. Additionally, our complication rates are comparable to the current experience of $6.8 \%$ to $22 \%$ reported in the literature $[1,2,10,15-18]$. Powers et al. [15] also demonstrated a significant decrease in the complication rate with conservative management, without an increase in length of hospital stay. This study did not provide data regarding hospital costs, as ours has. In addition, a 33\% failure rate of conservative management observed in their study is in striking contrast to our experience of no treatment failures.

Our data are in contrast to those of Bennion and Thompson [1], who could find no benefit associated with conservative management, but a significant increase in length of hospital stay. Our average length of hospital stay for both early, primary operative intervention and conservative management with interval appendectomy also fits well within the range found in the current literature $[2,5,10,13,17,18]$. As would be expected, there tends to be an increase in the length of hospital stay with increasing severity of disease $[1,5]$, with an overall decrease in length of stay over the past decade [16]. Additionally, our patients appear to have advanced to a regular diet earlier than those in the limited reports in the literature [17].

The ability to safely and accurately distinguish perforated from nonperforated acute appendicitis preoperatively is vital to the institution of conservative management measures. Clearly, the factor most predictive of perforated appendicitis is the duration of symptoms $[4,11,12]$. Age less than 6 years, high core temperature, significant leukocytosis, and evidence of a mass or diffuse peritonitis on physical examination have all been suggested as markers of perforated appendicitis [2], but the ability to distinguish perforated from nonperforated appendicitis solely on clinical examination remains inaccurate $[4,15]$.

High-resolution US with graded compression has proven to be a reliable technique for establishing the diagnosis of appendicitis [20-22]. Unfortunately, the effectiveness of this diagnostic tool is somewhat decreased in the setting of perforated appendicitis, perhaps due to abdominal wall rigidity with poor compression or the decompressed nature of the perforated appendix $[6,9,14]$. Despite this, we were successful at implementing a protocol of interval appendectomy for perforated appendicitis using available clinical and imaging techniques with excellent patient outcome.

In addition to the difficulties associated with errors in preoperative diagnosis, our study is retrospective in design and involves a relatively small number of patients. Also, during the period of time covered in this study, there was an overall gradual decrease in length of hospital stay. Despite this, the data suggest not only a decrease in the major complication rate with conservative management of perforated appendicitis, but just as importantly, management in a cost-effective manner with no significant increase in hospital stay or costs as compared with standard early operative intervention.

We believe that further efforts directed at determining the safety and utility of this form of management are justified. Obviously, systemic broad-spectrum antibiotics are responsible for the most significant decrease in mortality and morbidity seen in perforated appendicitis [10, 12], but all recent studies continue to document significant minor and major complication rates, with concern still raised regarding the occasional technical difficulties encountered in the course of appendectomy for perforated appendicitis [17]. There is clearly room for improvement in the management of perforated appendicitis. With the increasing constraints on health care resources, new management techniques and technologies should be asked to demonstrate cost-effectiveness in addition to safety and utility.

\section{References}

1. Bennion RS, Thompson JE Jr (1987) Early appendectomy for perforated appendicitis in children should not be abandoned. Surg Gynecol Obstet 165: 95-100

2. Birken GA, Schropp RP, Boles ET, et al (1986) Discharge planning for children with perforated appendicitis. J Pediatr Surg 21: $592-595$

3. Dolgin SE, Beck AR, Tartter PI (1992) The risk of perforation when children with possible appendicitis are observed in the hospital. Surg, Gynecol Obstet 175: 320-324

4. Gamal R, Moore TC (1990) Appendicitis in children aged 13 years and younger. Am J Surg 159: 589-592

5. Golladay SS, Sarrett JR (1988) Delayed diagnosis in pediatric appendicitis. South Med J 81: 38-42

6. Hayden CK Jr, Kuchelmeister J, Lipscomb TS (1992) Sonography of acute appendicitis in childhood: perforation versus nonperforation. J Ultrasound Med 11: 209-216

7. Hoffmann J, Rolff M, Lomborg V, et al (1991) Ultraconservative management of appendiceal abscess. J R Coll Surg Edinb 36: $18-20$

8. Jamick JS, Ein SH, Shandling B, et al (1980) Nonsurgical management of appendiceal mass in late presenting children. J Pediatr Surg 15: 574-576

9. Kao SCS, Smith WL, Abu-Yousef MM, et al (1989) Acute appendicitis in children: sonographic findings. AJR Am J Roentgenol 153: $375-379$

10. Karp MP, Caldarola VA, Cooney DR, et al (1986) The avoidable excesses in the management of perforated appendicitis in children. J Pediatr Surg 21: 506-510

11. Law WY, Fan ST, Yip WC, et al (1987) Acute appendicitis in children. Aust N Z J Surg 57: 927-931 
12. Linz DN, Hrabovsky EE, Franceschi D, Gauderer MWL (1993) Does the current health care environment contribute to increased morbidity and mortality of acute appendicitis in children. J Pediatr Surg 28: 321-328

13. Neilson IT, Laberge J-M, Nguyen LT, et al (1990) Appendicitis in children: current therapeutic recommendations. J Pediatr Surg 25: $1113-1116$

14. Ooms HWA, Koumans RKJ, Ho Kang You PJ, et al (1991) Ultrasonography in the diagnosis of acute appendicitis. Br J Surg 78: $315-318$

15. Powers RJ, Andrassy JR, Brennan LP, et al (1981) Alternate approach to the management of acute perforating appendicitis in children. Surg Gynecol Obstet 152: 473-475

16. Putnam TC, Gagliano NG, Emmens RW (1990) Appendicitis in children. Surg Gynecol Obstet 170: 527-532
17. Samelson SL, Reyes HM (1987) Management of perforated appendicitis in children --revisited. Arch Surg 122: 691-696

18. Schwartz MZ, Tapper D, Solenberger RI (1983) Management of perforated appendicitis in children: the controversy continues. Ann Surg 197: 407-411

19. Shipsey MR, O'Donnell B (1985) Conservative management of appendix mass in children. Ann R Coll Surg Engl 67: 23-24

20. Siegel MJ, Carel C, Surratt S (1991) Ultrasonography of acute abdominal pain in children. JAMA 266: 1987-1989

21. Skaane P, Amland PF, Nordshus T, et al (1990) Ultrasonography in patients with suspected acute appendicitis: a prospective study. $\mathrm{Br}$ J Radiology 63: 787-793

22. Wade DS, Morrow SE, Balsara ZN, et al (1993) Accuracy of ultrasound in the diagnosis of acute appendicitis compared with the surgeon's clinical impression. Arch Surg 128: 1039-1046 